Collection SFN 11 (2010) 89-100

(C) Owned by the authors, published by EDP Sciences, 2010

DOI: $10.1051 / \mathrm{sfn} / 201011006$

\title{
Réflectivité de neutrons : exemples d'études des systèmes en matière molle
}

\author{
G. Fragneto
}

Institut Laue-Langevin, 6 rue Jules Horowitz, BP. 156, 38042 Grenoble, France

\section{INTRODUCTION}

La réflectivité de la lumière sur une surface est un phénomène bien connu et provoqué par le changement d'indice de réfraction au passage à travers l'interface. Déjà en 1675, Newton avait observé que la couleur d'une couche mince de film de savon illuminé par un faisceau parallèle de lumière blanche, pouvait être utilisée pour estimer l'épaisseur de la couche. Les couleurs produites sont le résultat de l'interférence entre la lumière réfléchie en dessous et au-dessus des surfaces de la couche. L'interférométrie optique est encore utilisée aujourd'hui pour mesurer l'épaisseur de couches sur des surfaces.

Dans les années 20, des expériences menées par Compton ont démontré que la réflexion des rayons $\mathrm{X}$ est gouvernée par les mêmes lois que la réflexion de la lumière avec des indices de réfraction dépendant du nombre d'électrons par unité de volume. En 1944, Fermi et Zinn ont mis pour la première fois en évidence la réflectivité de neutrons [1]. Encore une fois, les mêmes équations fondamentales de la réflectivité optique sont suivies, mais les indices de réfraction dépendent non seulement des noyaux des atomes présents mais aussi de leur pouvoir de diffusion. Une quantité appelée «longueur de diffusion » peut être définie pour chaque noyau et indique sa capacité à diffuser les neutrons. L'indice de réfraction d'un matériel dépend donc de la longueur de diffusion des noyaux qui le composent. Comme dans le cas de la lumière, la réflexion totale peut se produire quand les neutrons traversent deux milieux avec une différence d'indice de réfraction positive. Les indices de réfraction de la plupart des éléments constitutifs et caractéristiques de la matière condensée étant inférieurs à ceux de l'air ou du vide, la réflexion totale interne est observée plus couramment que la réflexion totale externe observée avec la lumière. L'angle critique de réflexion totale est tel que la réflectivité de neutrons d'une longueur d'onde donnée à partir d'une interface a une valeur de 1 aux petits angles puis décroît très rapidement aux grands angles. Fermi et Zinn ont observé la réflexion totale des neutrons thermiques au-dessous de l'angle critique. La mesure de l'angle critique de réflexion totale a constitué une méthode importante pour déterminer les longueurs de diffusion des noyaux. Pendant longtemps, la réflectivité des neutrons a été utilisée non seulement pour le transport des neutrons thermiques dans les guides de neutrons, mais aussi pour ces déterminations, même si la présence d'inhomogénéités aux interfaces réduisait la précision des mesures. Lors de ces vingt dernières années, des méthodes plus précises ont été développées pour mesurer les longueurs de diffusion, et la réflectivité des neutrons est devenue une technique puissante pour étudier les inhomogénéités à travers les interfaces mêmes, inhomogénéités dues soit à la composition [2] soit à l'aimantation [3]. Comme dans le cas de la lumière, les ondes réfléchies au dessus et en dessous d'une couche peuvent interférer, ce qui provoque la formation de franges d'interférence dans le profil de réflectivité. Pour les études aux interfaces, la réflectivité des neutrons offre plusieurs avantages par rapport à des techniques optiques plus traditionnelles. En effet, à cause des petites longueurs d'onde des neutrons, la technique a une résolution de l'ordre de la fraction de nanomètre. Elle offre également des avantages par rapport aux rayons $\mathrm{X}$ car elle ne provoque pas

This is an Open Access article distributed under the terms of the Creative Commons Attribution-Noncommercial License 3.0, which permits unrestricted use, distribution, and reproduction in any noncommercial medium, provided the original work is properly cited. 
d'endommagement des échantillons et peut être utilisée pour étudier des interfaces cachées du fait de la faible absorption des neutrons qui pénètrent fortement dans la matière. La technique présente aussi l'avantage de permettre l'utilisation de la substitution isotopique pour atteindre des grands contrastes en densité de longueur de diffusion. L'information sur les profils de concentration, qui sont à l'origine des courbes de réflectivité, est obtenue en affinant des modèles théoriques. Les inhomogénéités latérales ne peuvent pas être étudiées par réflectivité spéculaire et on doit alors recourir à des études de diffusion hors spéculaire [4]. Ce dernier type d'études s'est beaucoup développé récemment et c'est certainement dans cette direction qu'il faut rechercher les potentialités futures de la technique.

La réflectivité des neutrons est utilisée pour des études de chimie de surface (tensioactifs, polymères, lipides, protéines et mélanges adsorbés aux interfaces liquide-fluide et solide-fluide), magnétisme des surfaces (couches ultrafines de Fer, multicouches magnétiques,) et couches solides (couches de Langmuir-Blodgett, couches minces solides, multicouches, couches de polymères).

Ce manuscrit présente une introduction sur la théorie et les méthodes de mesure de la réflectivité de neutrons avec une attention particulière portée sur les applications dans le domaine de la matière molle.

\section{PRINCIPES THÉORIQUES}

Deux approches de la théorie de la réflectivité de neutrons sont possibles. La première repose sur l'aspect ondulatoire des neutrons et est par conséquent similaire aux théories classiques d'optique sur le rayonnement lumineux [5]. La seconde traite les neutrons comme des particules qui sont décrites par une superposition linéaire d'ondes planes, solutions de l'équation de Schrödinger. La première approche est préférable dans le cas de l'analyse de la réflectivité spéculaire à cause de l'existence d'algorithmes permettant de calculer exactement la réflectivité de n'importe quelle structure normale à la surface ainsi que de solutions exactes et approchées de profils d'interfaces particuliers. En ce qui concerne la réflectivité non-spéculaire, il vaut mieux résoudre l'équation de Schrödinger. Une description plus compréhensible du neutron comme particule et de l'onde réfléchie associée a été proposée par Lekner [6]. L'équation de Schrödinger s'écrit :

$$
-\frac{h^{2}}{8 \pi^{2} m_{n}} \nabla^{2} \Psi+V \Psi=E \Psi
$$

où $h$ est la constante de Planck, $m_{n}$ la masse du neutron, $V$ le potentiel auquel est soumis le neutron et $E$ son énergie. $V$ représente les effets d'interactions entre le neutron et les diffuseurs du milieu qu'il traverse. Dans le cas ou le faisceau diffusé ne remplit pas la condition de Bragg de la structure cristalline traversée, le potentiel $V$ est considéré comme constant et uniquement fonction d'une longueur de diffusion cohérente (pseudo-potentiel de Fermi). Il s'écrit :

$$
V=\frac{h^{2}}{2 \pi m_{n}} N_{b}
$$

où $N_{b}$ est la densité de longueur de diffusion définie par

$$
N_{b}=\sum_{j} b_{j} n_{j}
$$

avec $n_{j}$ le nombre de noyaux par unité de volume et $b_{j}$ la longueur de diffusion du noyau $j$. Cette dernière est une quantité empirique, connue pour la plupart des noyaux. Comme déjà mentionné dans le cours d'introduction de J. Teixeira (ce livre), cette quantité varie de manière aléatoire à travers la classification périodique des éléments, et varie aussi surtout en fonction des isotopes d'un même élément. La valeur de $b$ de la plupart des matériaux est positive.

Considérons maintenant un faisceau de neutrons approchant une surface, de potentiel en volume $V_{o}$, infiniment profond (voir Figure 1). 

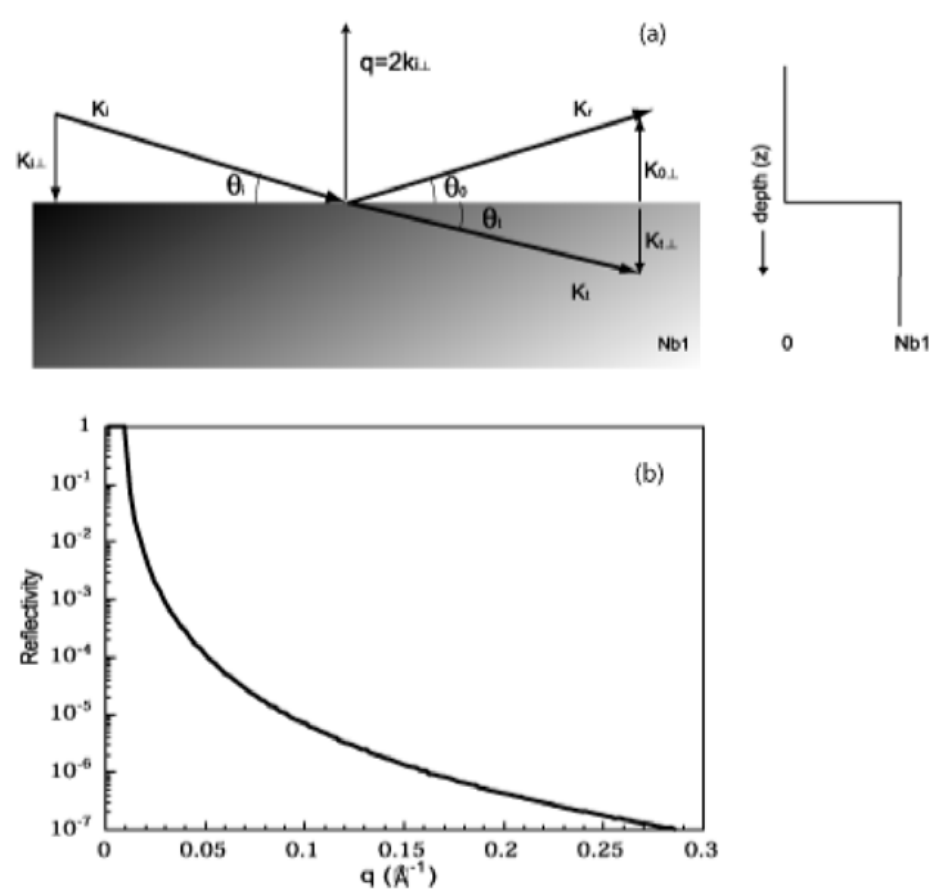

Figure 1. (a) Réflectivité d'un faisceau incident par une surface plane idéale. ki et kf sont les vecteurs d'onde incident et diffus, avec angles $q \mathrm{i}=\mathrm{qo}=\mathrm{q}$ dans le plan d'incidence; $q$ est le vecteur d'onde de transfert; Nb1 est la densité de longueur de diffusion du substrat. À droite, le profil de densité de longueur de diffusion en fonction de la profondeur. (b) Simulation de la réflectivité spéculaire de neutrons en fonction de $q$ pour une interface silicium/air $\left(\mathrm{Nb} 1=2.07 \times 10^{-6} \AA^{-2}\right)$.

S'il n'y a pas de structure le long de la surface, le seul gradient de potentiel, et par conséquent la force, est perpendiculaire à la surface. Seule la composante normale du vecteur d'onde incident, $k_{i}$, sera modifiée par la barrière de potentiel, et c'est la composante normale de l'énergie cinétique $E_{i \perp}$ qui détermine si le neutron est totalement réfléchi ou non.

$$
E_{i \perp}=\left(\frac{\left(h k_{i} \sin \theta_{i}\right)^{2}}{4 \pi m_{n}}\right)
$$

où $\theta_{i}$ est l'angle entre le faisceau incident et la surface.

Lorsque $E_{i \perp}<V_{o}$, il y a réflexion totale. La valeur du vecteur de diffusion critique, $\mathrm{q}_{c}$ pour $E_{i \perp}=V_{o}$ est donnée par:

$$
q_{c}=\sqrt{16 \pi N_{b}} \quad \text { et } \quad q=2 k_{i} \sin \theta_{i} .
$$

En supposant que la diffusion soit élastique, la condition de conservation des moments implique $\theta_{i}=\theta_{o}$ où $\theta_{o}$ est l'angle du faisceau réfléchi, i.e. la réflexion est spéculaire. Toute réflexion non spéculaire doit être le résultat de gradients de potentiel dans le plan $x y$ de la surface.

Lorsque $E_{i \perp}>V_{o}$, la réflexion n'est plus totale et le neutron peut être soit réfléchi soit transmis à l'intérieur du matériau. Le faisceau transmis $k_{t}$, avec la composante normale de l'énergie cinétique réduite par le potentiel, doit changer de direction, i.e. le faisceau est réfracté. Le changement du vecteur d'onde normal vaut :

$$
k_{t \perp}^{2}=k_{i \perp}^{2}-4 \pi N_{b} \text { ou } q_{t}^{2}=q_{i}^{2}-q_{c}^{2} .
$$


On définit alors un indice de réfraction relatif $n$ :

$$
n^{2}=\frac{k_{t}^{2}}{k_{i}^{2}}=\frac{k_{i / /}^{2}+\left(k_{i \perp}^{2}-4 \pi N_{b}\right)}{k_{i}^{2}}=1-\frac{4 \pi N_{b}}{k_{i}^{2}}=1-\frac{\lambda^{2} N_{b}}{\pi}
$$

où $\lambda$ est la longueur d'onde du neutron. Pour la plupart des matériaux, $\mathrm{N}_{b}$ étant beaucoup plus petit que 1 , une approximation de l'équation (7) conduit au résultat bien connu :

$$
n \approx 1-\frac{\lambda^{2} N_{b}}{2 \pi}
$$

On retrouve encore ici la remarque concernant le changement de longueur d'onde en volume est contraire à celui obtenu avec la diffusion de lumière (pour $b$ positif): $n$ est généralement plus petit que 1. Le faisceau transmis est donc réfracté à travers le plan du miroir, et à l'angle exact de réflexion totale, le faisceau est réfracté le long de la surface.

Tout le formalisme précédent (à l'exception du traitement des neutrons comme des ondes) peut être obtenu à partir de la physique classique. Cependant, pour pouvoir décrire tous les aspects de la réflectométrie, on va devoir utiliser l'approche de la mécanique quantique.

En séparant les variables $\mathrm{x}$, y et $\mathrm{z}$ de l'équation de Schrödinger, la fonction d'onde associée au neutron près d'une surface s'écrit :

$$
\frac{\partial^{2} \Psi_{z}}{\delta z^{z}}+k_{\perp}^{2}=0 \quad \text { où } \quad k_{\perp}^{2}=\frac{2 m_{n}}{\hbar^{2}}\left(E_{i}-V\right)-k_{/ /}^{2} .
$$

Les solutions générales de cette équation au-dessus et en dessous de la surface sont :

$$
\Psi_{z}=e^{i k_{i \perp}}+r e^{-i k_{i \perp} z} \quad \text { et } \quad Y_{z}=t e^{i k_{t \perp z}}
$$

où $r$ et $t$ sont les amplitudes de diffusion des ondes réfléchie et transmise. Les conditions de continuité de la fonction d'onde à l'interface $(\mathrm{z}=0)$ donnent :

$$
1+r=t \quad k_{i \perp}(1-r)=t k_{t \perp}
$$

ce qui conduit directement aux coefficients classiques de Fresnel que l'on trouve en optique :

$$
r=\frac{k_{i \perp}-k_{t \perp}}{k_{i \perp}+k_{t \perp}} \quad \text { and } \quad t=\frac{2 k_{i \perp}}{k_{i \perp}+k_{t \perp}} .
$$

En réflectométrie, on mesure la réflectivité en fonction du vecteur de transfert $q$. En combinant les équations (5), (6) et (12), on peut relier la réflectivité $R$ à $q$ et $q_{c}$. Notez que la mesure effectuée est une intensité, donc le carré d'une amplitude.

$$
R=r^{2}=\left[\frac{q-\left(q^{2}-q_{c}^{2}\right)^{1 / 2}}{q+\left(\left(q^{2}-q_{c}^{2}\right)^{1 / 2}\right)}\right]
$$

Lorsque $q \gg q_{c}$, l'équation précédente est réduite à :

$$
R \approx \frac{16 \pi^{2}}{q^{4}} N_{b}^{2}
$$

ce qui est la formule de la réflectivité utilisée dans l'approximation de Born [7].

Si on revient à la fonction d'onde à l'intérieur de la surface (10), on trouve en utilisant l'équation (6) que, lorsque $E_{I}<V$ (ou $k_{i \perp}^{2}<4 \pi N_{b}$ ou $q<q_{c}$ ), une solution réelle donnée par :

$$
Y_{z}=t e^{i\left(k_{i \perp}^{2}-4 p N_{b}\right)^{1 / 2} z}=t e^{-\frac{1}{2}\left(q_{c}^{2}-q^{2}\right)^{1 / 2} z} .
$$

Ce résultat est très important car il montre que lorsque la barrière de potentiel est plus grande que l'énergie de la particule normale à la surface, l'onde peut encore pénétrer sur une profondeur 

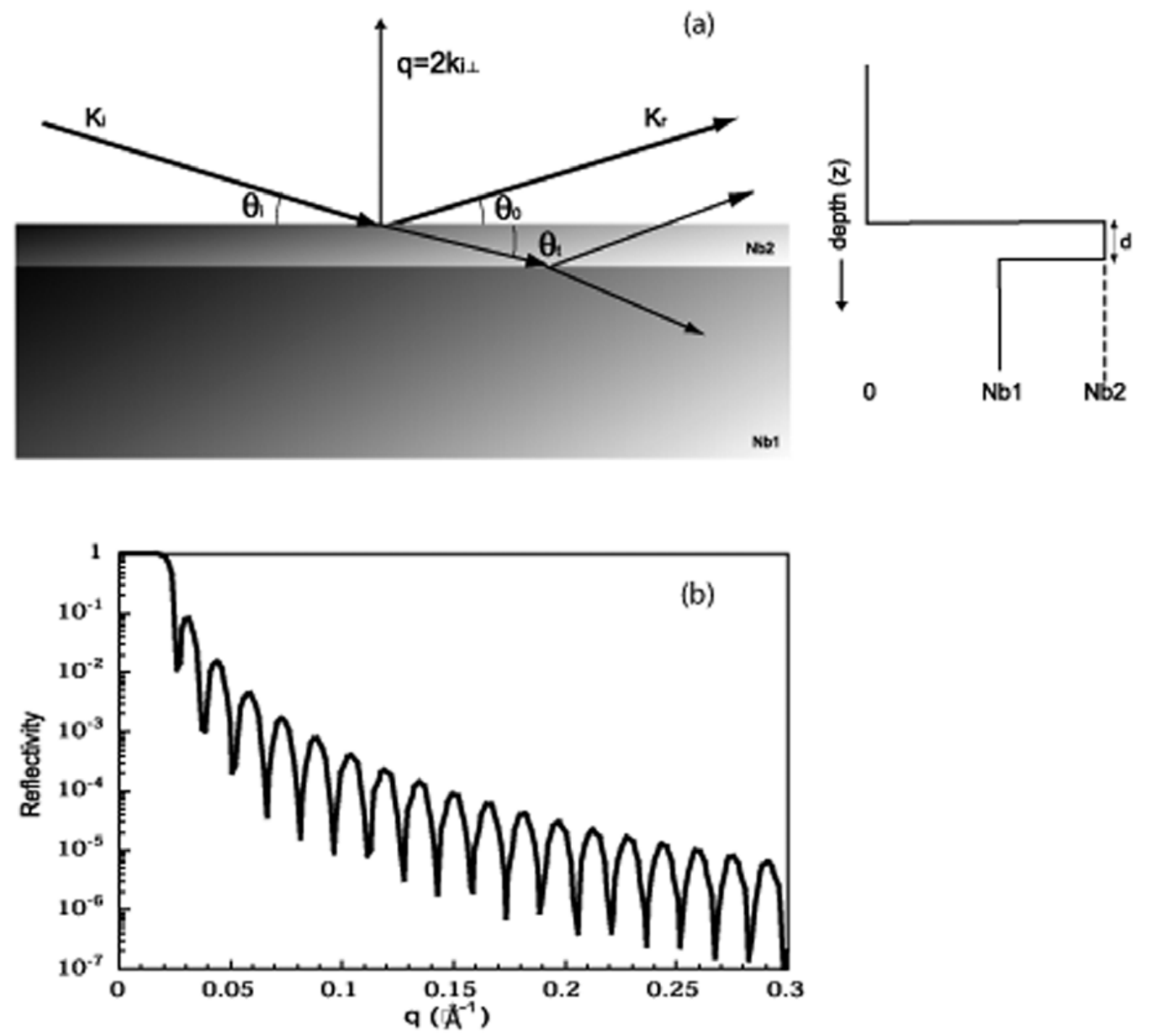

Figure 2. (a) Voir légende Figure 1(a) avec une couche additionnelle à la surface de densité de longueur de diffusion $\mathrm{Nb} 2$. (b) Simulation de la réflectivité spéculaire de neutrons en fonction de $q$ pour une couche de nickel d'épaisseur de $40 \mathrm{~nm}$ sur un substrat de silicium $\left(\mathrm{Nb} 2=9.04 \times 10^{-6} \AA^{-2}\right)$.

caractéristique de $\left(q_{c}^{2}-q^{2}\right)^{-1 / 2}$. Cette onde évanescente se propage le long de la surface avec un vecteur d'onde $k_{/ /}$et ressort ensuite du volume dans la direction spéculaire (voir figure 2). Par exemple, pour la valeur de $N_{b}$ de $\operatorname{Si}\left(2.07 \times 10^{-6} \AA^{-2}\right)$, la profondeur de pénétration est de l'ordre de $100 \AA$ à $q=0$; elle augmente rapidement à l' $\infty$ à $q=q_{c}$. Aucune règle de conservation n'est brisée, la réflectivité vaut 1 car cette onde ne représente aucun flux transmis au matériau. Ce résultat explique aussi pourquoi pour une couche mince $\left(<100 \AA\right.$ ) d'un matériau tel que $\mathrm{Ni}$, qui a une valeur de $\mathrm{N}_{b}$ deux fois plus grande que celle de $\mathrm{Si}$, déposée sur un substrat $\mathrm{Si}$, on retrouve une valeur de $\mathrm{q}_{c}$ définie par la valeur de $\mathrm{N}_{b}$ du $\mathrm{Si}$ et non par celle de Ni. Dans cet exemple, la couche est en fait plus petite que la longueur de pénétration caractéristique de l'onde plane.

Pour généraliser le calcul de la réflectivité à un système multicouches, on utilise souvent le formalisme matriciel utilisé en optique [7]. Les transmissions et réflexions entre une couche et la suivante peuvent être obtenues par le produit de matrices correspondant à chaque couche. Le problème qui consiste à inverser la courbe de réflectivité pour obtenir $N_{b}$ en fonction de la profondeur est compliqué car plusieurs profils peuvent conduire à la même courbe expérimentale. Les diverses techniques d'inversion utilisées peuvent être trouvées dans la revue [8]. 

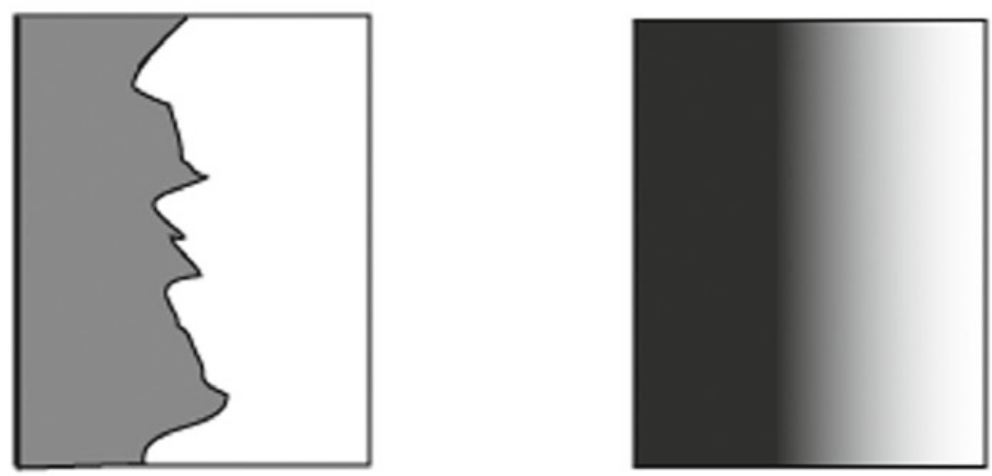

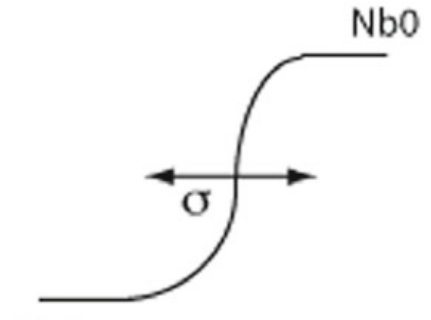

$\mathrm{Nb} 1$

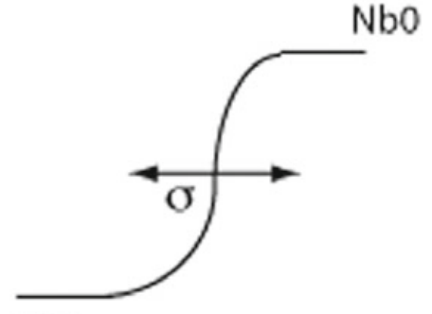

$\mathrm{Nb} 1$

Figure 3. Deux interfaces possibles qui peuvent générer le même profil de réflectivité spéculaire. Dans le cas de l'interface rugueuse, la diffusion hors-spéculaire est observée.

De nombreuses expériences de réflectivité visent à étudier les interfaces entre plusieurs matériaux. Une interface peut être rugueuse avec des aspérités et des creux sur des grandes échelles de distance, parfois avec une structure de type fractale. Il peut aussi exister une rugosité d'origine magnétique, lorsque l'aimantation ne change pas brutalement à l'interface (voir Figure 3). De même, une interdiffusion de matériaux va produire des interfaces imparfaites. On se rend compte que pour tous ces types d'interfaces, rugueuses ou diffuses, la réflectivité spéculaire est réduite par un facteur de type Debye-Waller [10]. L'équation (14) s'écrit

$$
R \approx\left(\frac{16 \pi^{2}}{q^{4}} N_{b}^{2}\right) e^{-q_{z}^{2} \sigma^{2}}
$$

où $\sigma$ caractérise la taille des imperfections de la couche. Que devient l'intensité perdue par ce facteur exponentiel? Dans le cas de l'interface diffuse, cette intensité va dans le faisceau transmis puisqu'il n'y a pas de gradient de potentiel dans une direction autre que celle normale à la surface. Par contre, dans le cas de l'interface rugueuse, l'intensité est perdue par des réflexions locales dans des directions autres que la direction spéculaire: c'est la diffusion hors spéculaire. Un paramètre comme la fonction de corrélation de hauteur peut être obtenu par des mesures de diffusion hors spéculaire [7, 11].

\section{MESURE DE LA RÉFLECTIVITÉ DE NEUTRONS}

Les composantes principales d'une expérience de réflectivité, que ce soit dans le cas de la lumière, des rayons $\mathrm{X}$ ou des neutrons, sont (i) une source de radiation, (ii) un sélecteur de longueur d'onde 


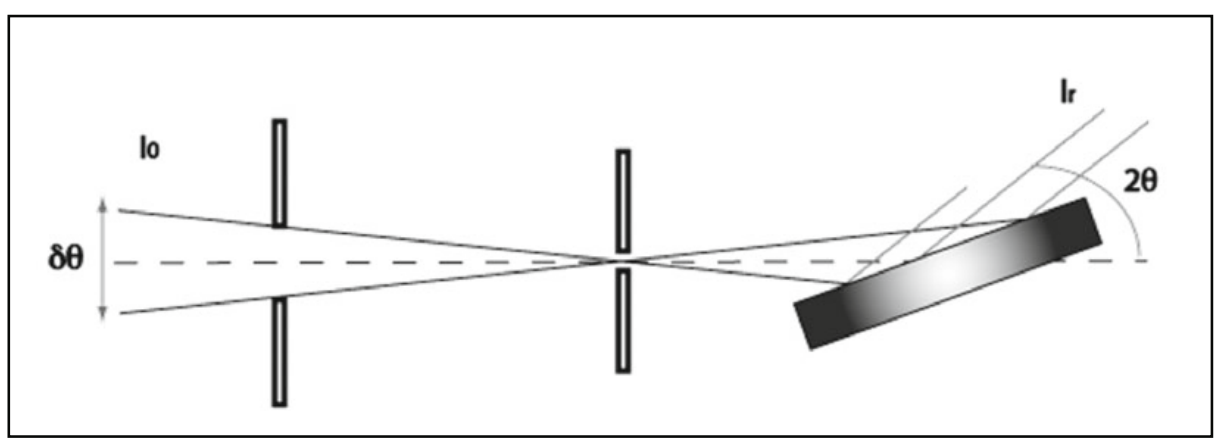

Figure 4. Une configuration de collimation typique dans le plan de réflexion. Quand l'échantillon est sous-illuminé (bruit de fond minimal), la distance entre la dernière fente et échantillon doit être la plus petite possible. En général, la divergence en dehors du plan de réflexion est définie seulement par la source.

(monochromateur, choppers), (iii) un système de collimation, (iv) l'échantillon et (v) un système de détection.

Les neutrons sont produits soit par un réacteur nucléaire où un faisceau continu est produit par fission nucléaire, soit par une source à spallation où un faisceau pulsé est obtenu quand un paquet de protons ou électrons de haute énergie circulant dans un accélérateur sont envoyés dans le noyau d'éléments lourds. Dans les deux cas, les énergies des neutrons sont trop élevées pour des études structurales ou en dynamique et elles sont réduites dans des modérateurs où les neutrons sont diffusés un grand nombre de fois et perdent de l'énergie à chaque collision jusqu'à ce que l'équilibre thermique soit atteint. Les énergies thermiques sont de l'ordre de $k_{B} T$ où $T$ est la température de l'eau du modérateur et $k_{B}$ est la constante de Boltzmann.

Le but d'une expérience de réflectivité spéculaire de neutrons est la mesure de la réflectivité en fonction du vecteur d'onde perpendiculaire à la surface réfléchissante, $q$. La mesure peut-être faîte soit en changeant l'angle d'incidence, $\theta$, soit la longueur d'onde $\lambda$, des neutrons, soit les deux. Dans la plupart des réacteurs nucléaires, les mesures sont normalement faites à une valeur fixée de $\lambda$, en utilisant des neutrons de grande longueur d'onde et des scans $\theta-2 \theta$ (angle du détecteur). La sélection de la longueur d'onde peut être obtenue par diffusion de Bragg d'un cristal monochromateur ou par sélection de vitesse à travers un disque mécanique. L'intensité incidente doit être aussi mesurée, soit en même temps que l'intensité réfléchie avec l'utilisation d'un second détecteur (moniteur), soit par une mesure complémentaire. La réflectivité est simplement le rapport de ces deux intensités pour chaque angle $\theta$ qui est converti en $q$ par la loi de Bragg :

$$
q=4 \pi \sin (\theta) / \lambda .
$$

Avec un échantillon de longueur de quelques centimètres et un angle initial $\theta$ d'une fraction de degré, le faisceau doit être bien collimaté pour s'assurer que l'échantillon est sous-illuminé, i.e que tout le faisceau incident touche la surface réfléchissante. Les petits échantillons peuvent être sur-illuminés, mais les données doivent être corrigées car le flux incident sur l'échantillon change quand $\theta$ augmente (voir Figure 4).

Le signal de réflectivité décroît très vite au-delà de l'angle critique quand $q$ augmente (voir (14), $\mathrm{R} \propto 1 / \mathrm{q}^{4}$ ). Pour gagner de l'intensité, les fentes de collimation peuvent être ouvertes, au prix d'une dégradation de la résolution, de façon à ce que $\delta \theta / \theta$ soit constant. $\delta \theta / \theta$ peut être choisi de telle sorte qu'il soit égal à la variation en longueur d'onde. La courbe de réflectivité finale doit avoir la résolution en $q, \delta q$, déconvoluée des données. La résolution en $q$ est liée a $\theta$ et $\lambda$ par la relation suivante :

$$
\left(\frac{\delta q}{q}\right)^{2}=\left(\frac{\delta \theta}{\theta}\right)^{2}+\left(\frac{\delta \lambda}{\lambda}\right)^{2}
$$


Cette méthode présente l'avantage que la longueur d'onde choisie peut correspondre au maximum de la distribution de neutrons de la source et, pour une résolution donnée, c'est la méthode la plus efficace d'utiliser le flux disponible.

En alternative, on peut utiliser le temps de vol («time-of-flight» ou TOF) et c'est cette option qui est le plus souvent utilisée dans les sources à spallation. Dans ce cas, on garde $\theta$ constant et on utilise toutes les longueurs d'onde présentes dans le faisceau incident. La longueur d'onde, et par conséquent $q$, est mesurée en pulsant le faisceau incident et en mesurant le temps d'arrivée au détecteur. La résolution est toujours donnée par (18) en replaçant $\delta \lambda / \lambda$ avec $\delta t / t$ où $\delta t$ est la largeur du temps du pulse et $t$ est le temps de vol du pulse. La gamme de $q$ couverte pour un $\theta$ donné dépend de la gamme de longueurs d'onde utile. Cette gamme est déterminée par la distribution des longueurs d'onde incidente, qui dépend de la température de thermalisation des neutrons et a une forme maxwellienne. Le flux aux longueurs d'onde maximale et minimale peut alors être inférieur de plus de deux ordres de grandeur à celui au maximum de la distribution. Pour la même résolution, la méthode TOF est moins efficace que la méthode monochromatique parce qu'elle demande un temps de comptage plus long pour mesurer la même gamme de q avec la même précision statistique. Par contre, elle est plus favorable pour des mesures de cinétique. Pour les expériences où on a un échantillon avec une structure à l'interface qui change en fonction du temps, seule la méthode TOF peut produire une $\mathrm{R}(\mathrm{q})$ unique à un temps donné. Les mesures $\theta-2 \theta$ sont des mesures séquentielles et chaque point en $q$ est mesuré a un temps différent. Par ailleurs, la méthode TOF est également très adaptée pour les mesures sur les liquides puisque sa géométrie est fixe. Dans la méthode TOF, pour résoudre le problème de réduction du flux, on peut utiliser un système de choppers (disques en rotation avec des fenêtres transparentes aux neutrons) pour changer la résolution en temps et par conséquent en longueur d'onde. La Figure 5 montre un exemple de réflectomètre qui opère en temps de vol. Il s'agit du réflectomètre FIGARO à l'ILL (Fluid Interfaces Grazing Angles ReflectOmeter) [12]. La gamme de longueurs d'onde utilisées est comprise entre $2 \AA$ et $30 \AA ̊$. Un système de quatre choppers, fonctionnant par couple de deux, permet de varier la résolution $(\Delta \lambda / \lambda)$ de $1.2 \%$ jusqu'à $10 \%$. Cette caractéristique est très intéressante pour les études en matière molle puisque les mesures avec des couches fines ne nécessitent pas de haute résolution et que, lorsque la résolution est diminuée, le flux de neutrons atteignant l'échantillon augmente. Un système de deux super-miroirs défléchit le faisceau entre $-4^{\circ}$ et $4^{\circ}$, ce qui permet ainsi d'arriver sur l'interface soit par le haut, soit par le bas. Le système de miroirs est suivi par un guide de collimation de deux mètres pour éliminer le bruit de fond dû aux réflexions hors spéculaires des supermiroirs. Des fentes très précises sont positionnées à l'entrée de ce guide et devant l'échantillon. Un détecteur bidimensionnel est placé à $2850 \mathrm{~mm}$ de l'échantillon et peut subir une rotation selon l'axe vertical pour détecter le signal réfléchi à tous les angles.

Lorsque $q$ augmente, le niveau de bruit augmente car certains neutrons ne sont pas réfléchis de façon spéculaire. La réflectivité est en effet très faible pour les grandes valeurs de $q$ et la plupart du faisceau traverse le substrat (par exemple l'eau) où elle est diffusée, soit de façon incohérente, soit par diffraction multiple. Une partie de ce faisceau diffusé arrive sur le détecteur à la même position que la réflexion spéculaire et contribue au bruit de fond, qui doit être soustrait du profil de réflectivité.

Il y a d'autres sources de bruit de fond en relation avec l'environnement de l'échantillon et des composantes du réflectomètre. Dans la plupart des cas, le signal de diffusion incohérente ${ }^{1}$ de l'échantillon même représente la source majeure de bruit. Autour du faisceau spéculaire réfléchi, il y a alors un signal de bruit de fond qui doit être soustrait avant de faire le rapport du faisceau réfléchi avec le faisceau incident. Ce bruit de fond peut être très important pour des expériences qui impliquent des interfaces avec l'eau. La réflectivité minimale qu'il est possible de mesurer est limitée dans ces cas à $\sim 10^{-7}$. En utilisant un détecteur bidimensionnel, on mesure sur une grande gamme d'angles $2 \theta$, ce

\footnotetext{
${ }^{1}$ Nous renvoyons le lecteur désireux d'en savoir plus sur l'origine de la diffusion incohérente aux articles de J. Teixeira et de R. Zorn de cet ouvrage.
} 


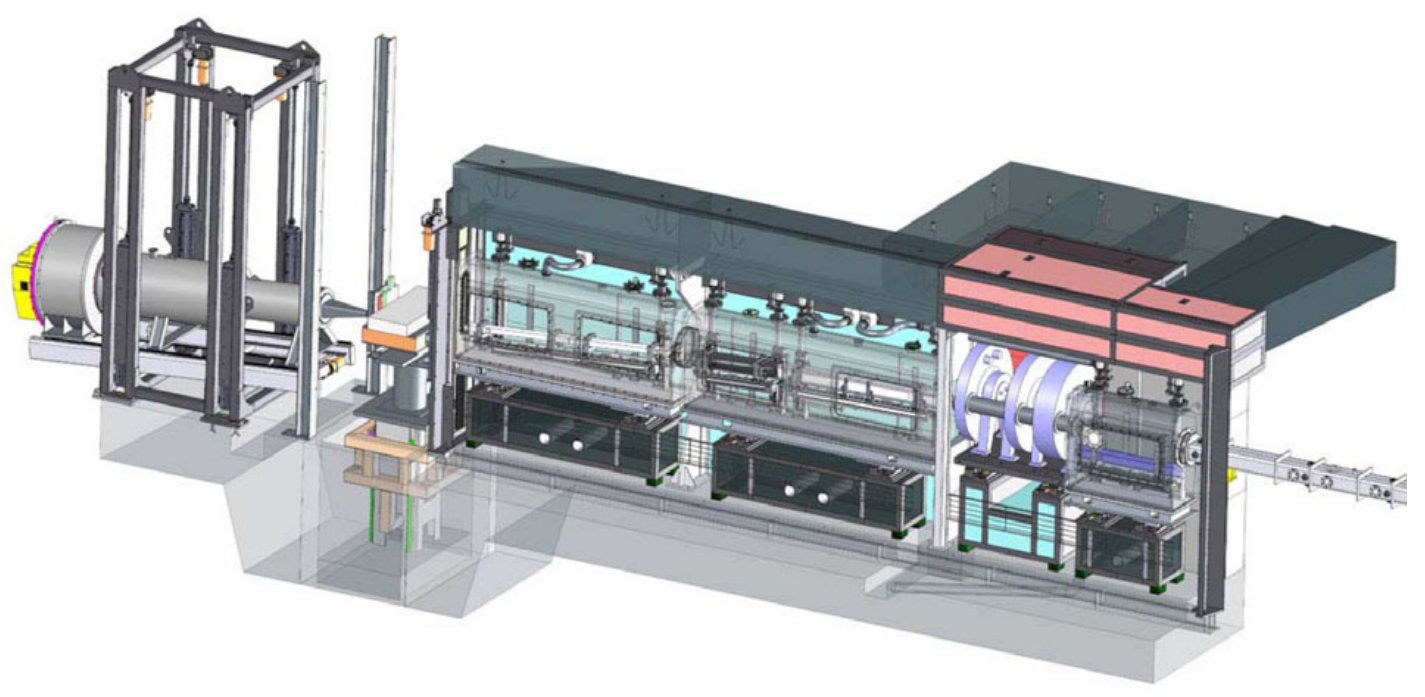

(a)

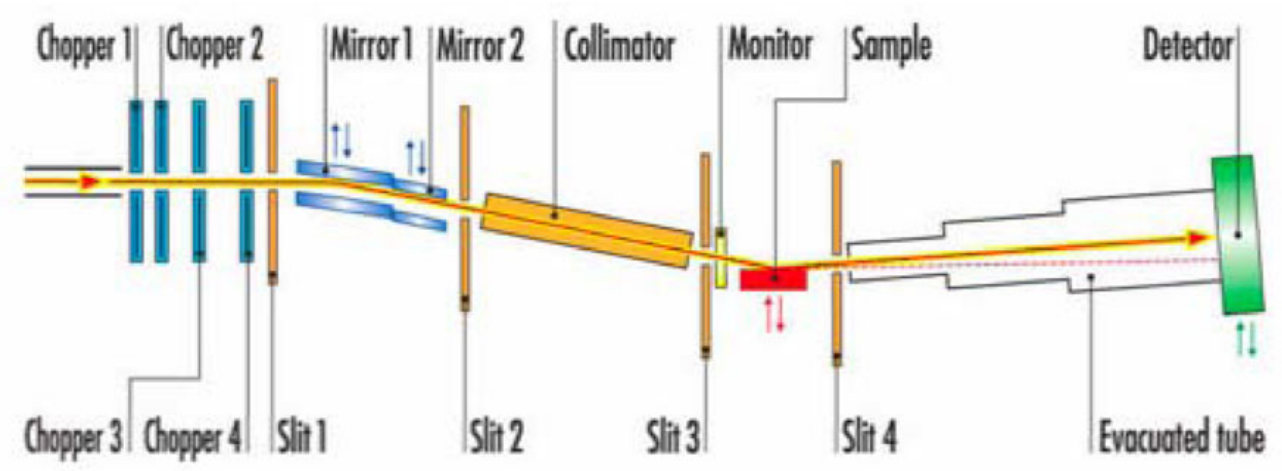

(b)

Figure 5. Le réflectomètre FIGARO à l'Institut Laue-Langevin [12]. (a) image 3-D de l'instrument positionné au bout du guide H171. (b) Schéma de l'instrument représentant les éléments principaux.

qui permet de mesurer le bruit de fond et la diffraction hors-spéculaire loin du faisceau réfléchi. Les instruments avec un détecteur ponctuel nécessitent une mesure séparée du signal hors-spéculaire. Dans les deux cas, il faut sélectionner avec attention la région en $2 \theta$ utilisée et s'assurer qu'elle ne contient pas d'intensité diffractée de l'échantillon même.

\subsection{Analyse des données}

La méthode d'analyse très souvent utilisée pour les données de réflectivité spéculaire implique la construction d'un modèle de l'interface constituée par une série de couches parallèles de matériel homogène. Chaque couche est caractérisée par une densité de longueur de diffusion, $\rho$, et une épaisseur, $d$, qui sont introduites pour calculer un profil de réflectivité modèle en utilisant la méthode de la matrice 
optique. La rugosité à l'interface entre deux couches consécutives peut être aussi incluse dans le modèle par la méthode d'Abeles [13]. Le profil calculé est comparé avec le profil mesuré et la qualité du fit vérifiée, soit en utilisant le $\chi^{2}$, soit la méthode de la moyenne quadratique. Les deux extrêmes de l'interface sont considérés avoir une épaisseur infinie et une densité de longueur de diffusion fixée. À l'interface, il y a soit une simple couche uniforme, soit une structure plus compliquée. En changeant $\rho$ ou $d$ pour chaque couche, le profil calculé peut être comparé avec le profil mesuré jusqu'à la détermination de l'affinement optimal des données. Chaque profil peut être décrit par plus d'un modèle. En matière molle, l'utilisation de différents contrastes isotopiques pour un système donné, associé aux contraintes physiques et chimiques du système, peuvent en général assurer la détermination d'un modèle unique. La variation de contraste est basée sur le fait que les noyaux diffusent les neutrons avec une amplitude différente et, parfois, comme dans le cas des protons et deutérons, avec une phase opposée. En utilisant un mélange de matériels hydrogénés et deuterés, le profil de réflectivité d'un système peut être changé en gardant la même structure à l'interface. Il est aussi possible en ajustant le rapport H/D de préparer des solvants ayant la même densité de longueur de diffusion que le substrat à travers lequel les neutrons passent avant d'arriver à l'interface, i.e. le solide pour une interface solide/liquide ou l'air pour les interfaces solide/air et liquide/air. Le contraste entre le solide (ou l'air) et le solvant devient nul et le profil de réflectivité ne dépend alors que de la région interfaciale. A partir de la valeur de la densité de longueur de diffusion à l'interface, on obtient des informations sur sa composition.

L'approximation cinématique [14] donne une description plus directe de la structure de monocouches solubles de tensioactifs à l'interface air/liquide. Récemment, Majkrzak et col [15] ont exploité une méthode sensible à la phase (phase-sensitive) pour révéler des profils de profondeur de la composition. Avec cette nouvelle méthode, le profil de densité de longueur de diffusion pour des membranes biomimétiques est obtenu à partir d'une inversion sans avoir besoin d'affiner ou d'ajouter de paramètres. Les autres approches qui n'utilisent pas de modèles se servent de l'entropie maximale [6] ou B-splines [17].

\section{RÉFLECTIVITÉ DE NEUTRONS ET MATIÈRE MOLLE}

Les applications en matière molle sont souvent les plus courantes pour la technique de réflectivité de neutrons. Les systèmes de la matière molle sont des systèmes complexes comme les polymères et élastomères, les cristaux liquides, les mousses, les tensioactifs, les lipides et les protéines. Ces systèmes sont importants autant dans le domaine de la biologie que pour la synthèse de nouveaux matériaux, la technologie de la nutrition, la récupération du pétrole et dans les nouvelles biotechnologies comme l'envoi ciblé de médicaments dans les cellules.

Il a déjà été évoqué que la réflectivité spéculaire des neutrons permet d'obtenir la distribution des indices de réfraction ou de densités de longueur de diffusion perpendiculaire à une surface ou interface, ce qui est en relation directe avec la composition ou le profil de concentration dans la région de l'interface. La géométrie en incidence rasante $\left(\theta\right.$ varie typiquement de $\sim 0.5^{\circ}$ à $4^{\circ}$ ) et la gamme de longueurs d'onde des neutrons froids ( $\lambda$ varie typiquement entre $\sim 2 \AA$ et $\sim 25 \AA$ ) permettent de sonder les échelles intéressantes en matière molle et vont de $\sim 1 \mathrm{~nm}$ jusqu'à $\sim 400 \mathrm{~nm}$. Les pouvoirs de diffusion de l'hydrogène et du deutérium sont très différents et permettent de moduler la distribution d'indices de réfraction grâce à la substitution isotopique (échange H/D), sans modifier la chimie des systèmes. Cette possibilité de moduler le contraste est une caractéristique puissante et très utilisée car elle permet de mettre en évidence des parties spécifiques de l'interface, d'étudier l'interface d'une bicouche de polymère, ou de sélectionner des composants dans des mélanges complexes adsorbés aux interfaces. Les neutrons froids sont aussi très pénétrants et permettent l'étude d'interfaces cachées. Les études ne sont pourtant pas limitées aux interfaces air-liquide et air-solide, mais peuvent être menées aussi aux interfaces solide-solide, solide-liquide et liquide-liquide. Récemment, les techniques de réflectivité avec une source de lumière synchrotron se sont imposées comme des techniques complémentaires aux neutrons dans l'étude de ces interfaces $[18,19]$. La limitation de ce type de rayonnement est liée 


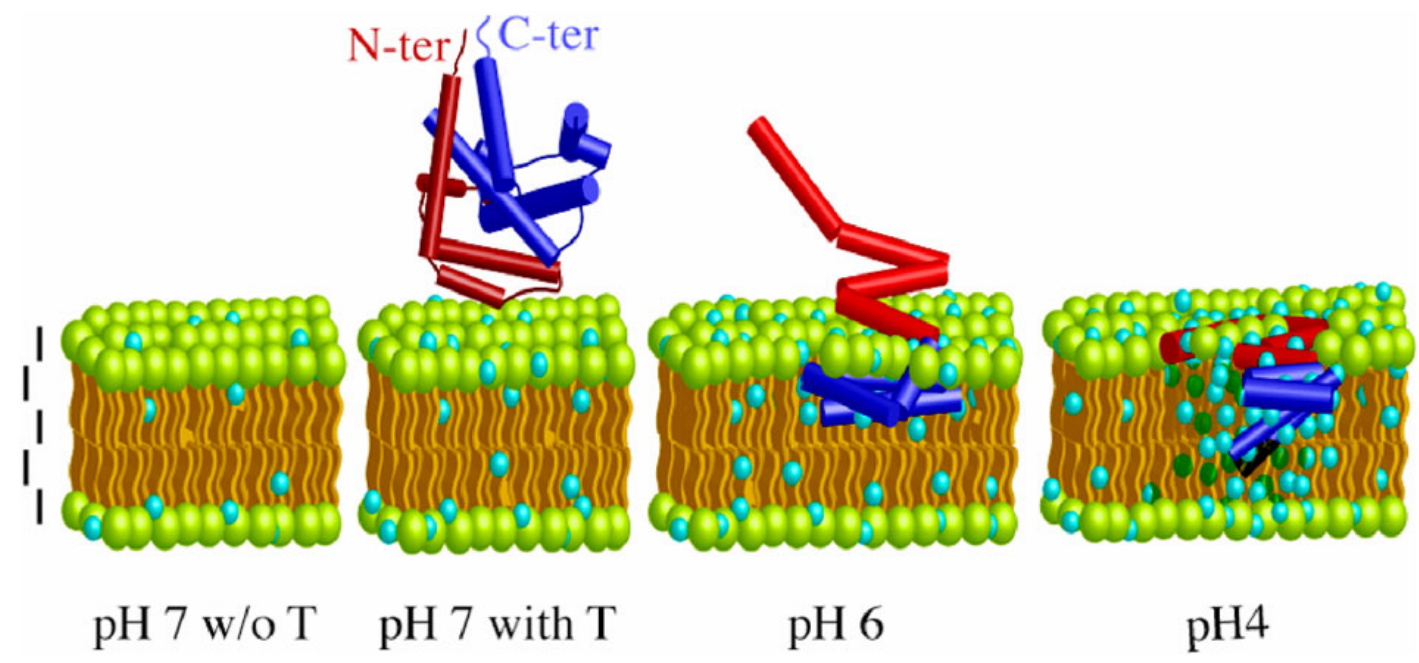

Figure 6. Schéma du mécanisme d'insertion membranaire du domaine de translocation de la toxine diphtérique obtenu à partir de mesures de RMN et réflectivité de neutrons en utilisant une protéine partiellement deuteriée (N-ter ou couleur rouge dans la figure). Figure reproduite à partir du manuscrit [24].

à l'endommagement des systèmes organiques par le faisceau, ce qui rend son utilisation bien moins pratique en matière molle par rapport à la réflectivité de neutrons.

Les nouvelles applications de la technique impliquent l'étude d'interfaces complexes comme celles des membranes biologiques, l'électrochimie «in-situ», l'adsorption aux interfaces liquidesolide et liquide-liquide, et des environnements complexes comme des surfaces sous cisaillement ou confinées. Concernant l'adsorption des polymères et tensioactifs, la tendance est d'étudier des structures complexes, des mélanges et le développement de nano-structures.

Des mesures d'électrochimie «in situ» sont décrites par exemple dans [20] où la mesure de la pénétration de solvants dans des films electro-polymerisés a été possible. Des mesures de fluides complexes confinés entre des surfaces de quartz et de saphir séparés par $\sim 100 \mathrm{~nm}$ sont décrites dans [21]. L'effet de l'épaisseur de couches de polymères à bloc immiscibles sur le degré d'interdiffusion à l'interface est décrit dans [22]. Ce dernier type d'études est décrit de façon exhaustive dans l'article de M. Sferrazza du présent ouvrage qui illustre les potentialités de la technique sur les interfaces de polymères.

L'étude de l'interface liquide-liquide mérite une mention particulière au vu de son importance technologique et de la difficulté de la sonder avec des techniques traditionnelles. Bowers et al. [23] ont établi une méthodologie de préparation qui consiste à créer une couche très fine d'huile entre un substrat de silice rendu hydrophobe et une solution aqueuse. Les neutrons traversent la silice pour atteindre l'interface.

A l'interface avec le domaine de la biologie, un exemple récent [24] montre l'importance de la deutériation pour améliorer la résolution de la technique. Le mécanisme d'insertion membranaire du domaine de translocation de la toxine diphtérique est élucidé grâce à des mesures de RMN et de reflectivité de neutrons en utilisant une protéine partiellement deutériée de façon à ce que sa présence dans la membrane soit plus facilement détectable. Une représentation schématique du mécanisme à des pH différents est montrée sur la Figure 6.

La réflectivité de neutrons reste pourtant une technique à basse résolution. Son exploitation optimale dépend de la possibilité de mener des études complémentaires sur les systèmes utilisés. 
Pour plus de détails, le lecteur peut consulter des revues récentes sur les études par réflectivité de neutrons en matière molle $[25,26]$ ou visant plus en particulier aux domaines des interfaces de polymères [27], la biologie [28, 29], les tensioactifs et mélanges polymères-tensioactifs [30, 31].

\section{Remerciements}

Un grand merci à Annie Brûlet et Fabrice Cousin pour leur aide dans la rédaction du texte en français.

\section{Références}

[1] Fermi, E. and Zinn, W. H. 1946, Phys. Rev. 70, 103.

[2] Penfold J. and Thomas R. K. 1990 J. Phys. Condens. Matter 21369

[3] Felcher G. P. 1981 Phys. Rev. B 241995

[4] Sinha S. K., Sirota E. B., Garoff S. and Stanley H. B. 1998 Phys. Rev. B 382297

[5] Sears V. F. 'Neutron Optics', Oxford Press, Oxford (1989)

[6] Lekner J. 1987 in: "Theory of Reflection” Martinus Nijhoff Dordrecht

[7] Born M. and Wolfe E. 1989 in: "Principles of Optics" Pergamon Press Eds. Oxford

[8] Zhou X.-L. and Chen S.-H. 1995 Phys. Rep.257 223

[9] Williams, W. 1989, Polarized Neutrons, Oxford Univ. Press, Oxford

[10] Névot L. and Croce P. 1990 Rev. de Phys. Appl. 15761

[11] Daillant J. and Gibaud A. 1999 in: "X-ray and Neutron Reflectivity: Principles and Applications" Springer Eds.

[12] http://www.ill.eu/instruments-support/instruments-groups/instruments/figaro/

[13] Heavens O. S. 1955 in: “Optical Properties of Thin Films”, Butterworths Eds. London

[14] Crowley T. L. 1993 Physica A 195354

[15] Majkrzak C. F., Berk N. F., Krueger S., Dura J. A., Tarek M., Tobias D., Silin V., Meuse C. W., Woodward J. and Plant A. 2000 L. Biophys. J. 793330

[16] Silvia D. S., Hamilton W. A. and Smith G. S. 1991 Physica B 173121

[17] Berk, N. F. and Majkrzak, C. F. 1991, Phys. Rev. B 51, 11, 296

[18] Daillant J., Mora S., Fradin C., Alba M., Braslau A. and Luzet D., Appl. Surf. Sci., vol. 182, issues 3-4, 223-230.

[19] Daillant D. J., Bellet-Amalric E., Braslau A., Charitat T., Fragneto G., Graner F., Mora S., Rieutord F. and Stidder B., Proc. Nat. Acad. Sci., 2005, 102 (33): 11639

[20] Cooper J. M., Cubitt R., Dalgliesh R. M., et al. J. Am. Chem. Soc. 126 (47): 15362-15363, 2004

[21] Kuhl T. L., Smith G. S., Isrealachivili J. N., Majewski J., Hamilton W., Rev Sci Inst 2001; 72: 1715.

[22] Carelli C., Jones R. A. L., Young R. N., Cubitt R., Dalgliesh R., Schmid F. and Sferrazza M., Phys. Rev. E 72, 031807 (2005)

[23] Bowers J., Zarbakhsh A., Webster J. R. P., Hutchings L. R., Richards R. W. Langmuir 2001;17:140.

[24] A. Chenal, L. Prongidi-Fix, A. Perier, C. Aisenbrey, G. Vernier, S. Lambotte, M. Haertlein, M.-T. Dauvergne, G. Fragneto, B. Bechinger, D. Gillet, V. Forge and M. Ferrand, J. Mol. Biol. (2009) 391, 872-883

[25] Penfold J. et al. 1997 J. Chem. Soc. Faraday Trans. 93(22) 3899

[26] Penfold J., Cur Opin Coll Int Sci Volume 7, Issues 1-2 , March 2002, Pages 139-147

[27] Sferrazza M., Jones R. A. L., Penfold J., Bucknall D. G., Webster J. R. P. J Mat Chem 2000;10:127.

[28] Krueger S. Cur Opin Coll Int Sci 2001;6:111.

[29] Fragneto-Cusani G. J Phys: Codens Matt 2001;13:4973.

[30] Lu J. R., Thomas R. K., Penfold J. Adv Con Int Sci 2000;84:143.

[31] Thomas R. K., Ann. Rev. Phys. Chem. 55: 391-426 2004 